\title{
COMPARAÇÃO DO DESEMPENHO DE CARTEIRAS UTILIZANDO OS MÉTODOS PARI- DADE DE RISCO, MÍNIMA VARIÂNCIA E EQUAL WEIGHTING: UM ESTUDO NO MER- CADO BRASILEIRO EM PERÍODOS PRÉ, DURANTE E PÓS A CRISE DE 2008
}

PORTFOLIO COMPARISON PERFORMANCE USING THE RISK PARITY, MINIMUM VARIANCE AND EQUAL WEIGHTING METHODS: A STUDY IN THE BRAZILIAN MARKET IN PERIODS BEFORE, DURING, AND AFTER THE CRISIS OF 2008

\author{
Maurício Mesquita Bortoluzzo ${ }^{2}$ \\ Doutor pela Universidade Presbiteriana Mackenzie \\ Saint Paul Escola de Negócios \\ mauriciomesquita@hotmail.com \\ https://orcid.org/0000-0002-7750-7411 \\ Adriana Bruscato Bortoluzzo \\ Doutora em Estatística - USP \\ INSPER (Instituto de Ensino e Pesquisa) \\ bruscato@uol.com.br \\ https://orcid.org/0000-0003-2872-031X
}

Ricardo Alexandre Imamura

Bacharelado em Economia - INSPER (Instituto de Ensino e Pesquisa)

ricardoai1@al.insper.edu.br https://orcid.org/0000-0002-2867-8476

Tatiana Terabayashi Melhado

Doutora em Estatística pelo Instituto de Matemática e Estatística da USP (IME-USP)

INSPER (Instituto de Ensino e Pesquisa) e ESPM (Escola Superior de Propaganda e Marketing)

tatianatm@insper.edu.br

https://orcid.org/0000-0003-1114-3026

\section{Rita de Cássia Marques Lima de Castro}

Pós-doutorado (Faculdade de Administração, Economia e Ciências Contábeis - FEA - USP).

Doutora em Ciências - (Programa de Pós-Graduação Interunidades em Integração da América Latina - PROLAM-USP) Universidade de São Paulo (USP)

Universidade de Mogi das Cruzes - UMC - Campus Villa-Lobos (SP) e pesquisadora vinculada a CORS-USP e NESPIUSP

ritalimadecastro@gmail.com

http://orcid.org/0000-0002-0137-6005

\footnotetext{
${ }_{1}$ Artigo recebido em: 18/10/2017. Revisado por pares em: 15/11/2017. Reformulado em: 15/02/2018. Recomendado para publicação, após a segunda rodada, em: 30/05/2018 por Luiz Felipe de Araújo Pontes Girão (Editor Geral). Publicado em: 04/07/2018. Organização responsável pelo periódico: UFPB.

2 Endereço: Rua da Consolação, n. 930, Consolação - SP, 01302-907

DOI: https://doi.org/10.22478/ufpb.2318-1001.2018v6n3.36822
} 


\section{RESUMO}

Objetivo: Comparação do desempenho de carteiras montadas de acordo com os métodos Paridade de Risco (PR), Mínima Variância (MV) e Equal Weighting (EW) no mercado brasileiro em períodos pré, pós e durante a crise internacional de 2008.

Fundamento: Seleção de carteiras por meio do método MV tem base no artigo de Markowitz (1952). O método de alocação EW, estudado por Choueifaty e Coignard (2008), é o único portfólio na fronteira eficiente no caso de todos os ativos possuírem a mesma correlação. Maillard, Roncalli e Teïletche (2010) apresentam a metodologia PR como a mais eficiente na alocação de ativos por equalizar os pesos de cada ativo de acordo com sua contribuição ao risco do portfólio.

Método: Foram construídas carteiras anuais com dados diários de todos os ativos brasileiros contemplando o período de 2007 a 2013. Foi utilizado o Índice de Sharpe (IS) para avaliar o desempenho das carteiras nos períodos anteriores, durante e após a crise.

Resultados: O tradicional método MV apresenta maiores valores de IS em aproximadamente 70\% das vezes. Já o método PR foi mais eficaz em períodos de crise.

Contribuições: A comparação do desempenho de diferentes metodologias na construção de carteiras de risco brasileiras em períodos com diferentes volatilidades é a principal contribuição do trabalho. $\mathrm{O}$ fato de que o método EW apresentou a pior relação risco retorno em praticamente todos os períodos da amostra enseja ao mercado a busca por metodologias mais sofisticadas de alocação em ativos de risco.

Palavras-chave: Alocação de carteiras. Mínima Variância. Equal Weighting. Paridade de Risco. Índice de Sharpe.

\section{ABSTRACT}

Objective: Portfolios Comparison performance are assembled according to the Risk Parity (RP), Minimum Variance (MV) and Equity Weighting (EW) in the Brazilian market in periods before, after and during the international crisis in 2008.

Background: Selection of portfolios using the MV method is based on the paper by Markowitz (1952). The EW allocation method, studied by Choueifaty and Coignard (2008), is the only portfolio at the efficient frontier in case all assets have the same correlation. Maillard, Roncalli and Teiletche (2010) presented the RP as a more efficient methodology by equalizing the weights of each asset according to its contribution to portfolio risk

Method: Annual portfolios with daily Brazilian data covering the period from 2007 to 2013 were constructed. The Sharpe Index (SI) is used to evaluate the performance of the charts in the before, during and after the crisis.

Results: The traditional MV method presents higher SI values in approximately $70 \%$ of the time. The PR method is the most effective in times of financial crisis.

Contributions: the comparison of the performance of different methodologies in the construction of Brazilian risk portfolios in periods with different volatilities is the main contribution of this paper. The fact that the EW method presented the worst risk-return relationship in practically all the periods of the sample leads to the market searching for more sophisticated methodologies for allocating risky assets.

Keywords: Portfolios Allocation. Minimum Variance. Equal Weighting. Risk Parity. Sharpe Index.

\section{INTRODUÇÃO}


Um dos principais objetivos presentes no dia a dia dos gestores de fundos de investimentos é criar um portfólio baseado na melhor remuneração para cada unidade de risco assumida (entendido neste contexto como a variância dos retornos). A teoria de alocação de portfólio (Portfolio Selection), formulada por Markowitz (1952), a qual consiste na seleção de carteiras por meio do método de média-variância, foi relevante para o desenvolvimento de métodos quantitativos para atingir este objetivo. Uma evidência dessa relevância encontra-se não somente no número de citações que a obra original de Markovitz apresenta, ultrapassando onze mil constâncias em artigos acadêmicos até finais de 2017 na plataforma Web of Science, mas em seu legado, fundamentando a moderna teoria sobre portfólio e reforçando a diversificação como o tema que se tornou a principal discussão na área de Finanças, como destacam Fabozzi, Gupta e Markowitz (2002) e Araújo e Montini (2015). Contudo, tal teoria apresenta alguns problemas com relação às premissas na formação da fronteira eficiente e aqui está um mote que levou pesquisadores a buscarem novos modelos.

No mercado financeiro é comum a utilização de dois métodos para alocação de ações: o Método de Mínima Variância (doravante denominado MV) e o Equal Weighting (denominado EW). Utilizado no mercado e originado com base na teoria de Portfolio Selection, o método de Mínima Variância (MV) se propõe a formar carteiras com o menor risco possível. Entretanto, o MV está sujeito às mesmas críticas feitas por Zhou (2009) ao método de média-variância. Para esse autor, quando nenhuma restrição é utilizada, a otimização gera uma alocação com pesos anormalmente altos, tanto para posições compradas (long) como vendidas (short), ou quando se impõe restrição a posições vendidas, baixa diversificação.

Outro método largamente utilizado para montagem das carteiras pensando na máxima diversificação é o chamado Equal Weighting (EW). Esse é um método simples de ser implementado por dar o mesmo peso para todos os ativos, ou seja, permite a máxima diversificação da carteira, mas, conforme exposto em Maillard et al.(2010), apresenta um maior risco total.

Cumpre ressaltar que, embora largamente utilizados, ambos os métodos possuem dois principais pontos negativos. O primeiro (MV) é decorrente da forma pelas quais as carteiras são pouco diversificadas. Enquanto o segundo (EW), por dar o mesmo peso para todos os ativos, tem a máxima diversificação, porém com maior risco total. Assim, os indivíduos atuantes no mercado continuam em sua busca por novos métodos que otimizem o portfólio de ações.

Com o objetivo de aumentar a diversificação da carteira em relação ao método MV e manter o risco total pequeno, com valor o mais próximo possível do método MV, Maillard, Roncalli e Teiletche (2010) propuseram a utilização de um método conhecido como Paridade de Risco (PR) para a formação das carteiras de investimento. Este método, menos encontrado em produções acadêmicas, diferencia-se em relação aos demais pela busca da equalização dos riscos com a maximização do retorno total, considerando-se um nível máximo de risco a ser atingido. Utilizando dados do Brasil, alguns dos estudos recentes sobre a aplicação de paridade de risco no mercado brasileiro foram realizados por Souza, Filomena, Caldeira, Borenstein e Righi (2017); Souza, Filomena e Caldeira (2015).

A comparação do desempenho de diferentes metodologias na construção de carteiras de risco no mercado de capitais brasileiro em períodos com diferentes volatilidades é a principal contribuição deste trabalho. Cumpre ressaltar que, embora haja outros trabalhos que realizam comparações de métodos, como os de Araújo e Montini (2015) ou estude a Paridade de Risco, como os já citados estudos de Souza et al. (2017) e Souza et al. (2015), bem como os realizados por Bortoluzzo Venezuela, Bortoluzzo e Nakamura (2016), a diversidade de resultados abre espaço para a discussão sobre métodos mais eficientes na otimização dos portfólios. Nesse sentido, a presente pesquisa busca contribuir para evidenciar a necessidade de o mercado continuar buscando metodologias mais sofisticadas de alocação de recursos em ativos de risco. 
Com relação à originalidade, o fato de lidar com todos os ativos líquidos do mercado brasileiro para composição das carteiras via Paridade de Risco é o que diferencia este trabalho dos demais encontrados no Brasil e no estrangeiro, o que gerou a necessidade de utilização de pacotes computacionais mais robustos, no caso o Matlab, para execução dos programas confeccionados pelos autores sem restrição na quantidade de ativos. Souza et al. (2017) e Souza et al. (2015), trabalhos que utilizam dados do mercado brasileiro com o método PR, abrangeram apenas sete índices setoriais (energia elétrica, industrial, consumo, imobiliário, financeiro, materiais básicos e utilidade pública) no período de 2008 a 2014, enquanto Chaves, Hsu, Li and Shakernia (2011) trabalharam com nove classes de ativos do mercado americano de 1980 a 2010, e Qian (2011) usou apenas dois tipos de investimentos (ativos e bonds) no mercado americano para explicar o funcionamento do método PR.

Desta forma, o objetivo deste trabalho é comparar o desempenho das carteiras formadas pelos três métodos, por meio do IS, para o período de 2007 a 2013, abrangendo o período de crise econômica ocorrido nos EUA, que se iniciou naquele país em 2007 com o estouro da bolha do subprime, e afetou diretamente o Brasil em 2008 após o estouro da bolha no mercado de commodities (Bortoluzzo et al., 2016). Foram considerados desempenhos no período total, além dos desempenhos anteriores, durante e após a crise. Para tanto, o trabalho está assim estruturado: na próxima seção é realizada a fundamentação teórica, a metodologia é descrita na seção 3, na seção 4 são analisados os resultados obtidos e a conclusão é feita na seção 5 .

\section{FUNDAMENTO}

Antes de adentrar na questão-chave deste trabalho, que é a comparação do desempenho de portfólios montados a partir de três tipos de métodos de estimação, considerando-se como marco temporal a crise econômica de 2008 no Brasil, cabe uma breve discussão sobre evidências empíricas que abordaram o tema de desempenho de portfólios brasileiros comparando-se distintos métodos de alocação de ativos e considerando períodos de crise.

Bortoluzzo et al. (2016), ao compararem modelos unifatorial (CAPM) e multifatoriais de risco (modelo de três fatores de Fama e French (1993) e o de quatro fatores de Carhart (1997)) no período de 2002 a 2013, identificaram que o modelo de Carhart, com maior número de fatores (quatro), foi o que teve melhor desempenho preditivo, mas um achado que chama a atenção em seus resultados é o de que esse ganho foi apenas marginal nos períodos antes da crise e na própria crise, sendo somente após a crise que o modelo apresentou um desempenho significativamente superior ao de Fama e French, apresentando mais de $40 \%$ de melhorias em seu caráter preditivo, em comparação ao modelo de três fatores.

Souza et al. (2017) e Souza et al. (2015), ao realizarem um estudo sobre a aplicação de paridade de risco no Brasil, identificaram, originalmente, que a maneira com que se estima a matriz de covariância não teve uma influência relevante ao estudo de alocação de carteiras mediante o método de paridade de risco. Sendo pioneiros na aplicação do estudo no mercado de ações brasileiro, Souza et al. (2015) destacaram as limitações de seus achados. No entanto, os estudos realizados corroboraram os achados realizados em outros países com a aplicação do método de paridade de risco. Nos estudos feitos, os autores encontraram que o método de paridade de risco concentra-se em uma escala intermediária entre o método de pesos iguais para os ativos (EW) e o método de mínima variância (MV). Os autores trabalharam com sete índices setoriais da B3 para a formação de carteiras como se estes índices fossem ativos.

Neste trabalho são utilizados os mesmos métodos dos trabalhos acima citados, mas com a contribuição de que todos os ativos com liquidez da B3 no período avaliado foram utilizados. Apesar da maior complexidade do procedimento de otimização de paridade de risco, isso mostra que o método pode ser implementado para um maior número de ativos à escolha do investidor, se assemelhando ao que é comumente feito no mercado. 
Levando em conta que se busca desenvolver modelos de alocação de ativos em portfólio visando alcançar uma composição de carteiras de ativos financeiros que apresente, sob o ponto de vista de um investidor, a melhor relação risco-retorno, é recomendável que o estudo desses modelos considere distintos cenários, inclusive o de pré-crise, crise e pós-crise. Assim, após a fundamentação sobre alocação de ativos e a descrição de cada método, faz-se uma análise do impacto desses métodos considerando os três momentos temporais: pré, durante e pós a crise econômica de 2008.

\subsection{Alocação de Ativos}

O retorno da carteira é a combinação ponderada da proporção do retorno dos ativos que a constituem e a volatilidade da carteira depende da correlação entre os ativos. Como apontado na seção anterior, à seleção de carteiras por meio do método MV, criado por Markowitz (1952), é a mais presente no dia a dia dos agentes econômicos (Levy \& Levy, 2014). Esse método é caracterizado pela busca da otimização da média-variância de um portfólio. A lógica está na busca da redução do risco, e essa redução é comumente conquistada ou mediante a diversificação das ações, conforme aponta a obra clássica de Markowitz (1952) e os trabalhos de Sharpe (1963; 1964), ou por meio da construção de uma carteira que fique balanceada (Ferri, 2010). O problema é formulado de modo a se minimizar o risco do portfólio para um dado nível de retorno requerido pelo investidor e/ou maximizar o nível de retorno esperado do portfólio, associado a um dado nível de risco.

O termo Fronteira Eficiente, utilizando pontos de risco-retorno gerados a partir da combinação entre ativos com e sem risco, advém dos estudos de Markowitz (1952) sobre a relação entre retorno, risco e correlação dos ativos, provando que, ao se diversificar entre ativos livres de risco (como títulos públicos), os que têm retornos com desvio padrão igual a zero, e ativos de risco (de empresas públicas ou privadas negociados na B3), é possível gerar uma melhor relação risco retorno do que uma carteira menos diversificada, embora possivelmente a custas de maiores custos de gestão.

Ao utilizar o retorno esperado fixo dos ativos para criar a fronteira eficiente e todas as possíveis combinações dos ativos, o modelo de Markowitz (1952) usa suposições quanto ao futuro, suposições estas feitas com base em incertezas (Rogers, Securato \& Ribeiro, 2007). Portanto, o maior problema do modelo está na questão da definição dos parâmetros usados na construção do portfólio serem fixos. Qualquer mudança nesses parâmetros, seja ela pequena ou grande, gerará mudanças significativas na composição da carteira (Maillard et al., 2010; Leal, Silva \& Ribeiro, 2002).

Considerando-se essa limitação, demais métodos foram criados em busca de maior coesão e menor incerteza (Chow, Hsu, Kalesnik \& Little, 2011). Fliege \& Werner (2014) realizam a otimização de portfólio para uma fronteira eficiente robusta, em que as restrições do modelo se mantêm válidas sob os possíveis valores que os retornos podem assumir, isto é, considerando a incerteza dos dados. Visando um balanceamento entre a redução do erro de amostragem e a perda de informações de amostra, Behr, Guettler e Miebs (2013) propuseram uma estratégia de portfólio de Mínima Variância com restrições flexíveis superiores e inferiores para os pesos da carteira. Ao calibrarem as restrições de peso da carteira, os autores conseguem equilibrar o resultado entre a perda de informações amostrais e o ganho na redução de erros amostrais, ambos decorrentes dessas restrições impostas. O resultado dessa calibragem foi a obtenção de maiores índices de Sharpe do que os resultantes de portfolios que foram compostos com ponderação igualitária ou Equal Weighting (EW). Nguyen e Lo (2012) afirmam que a otimização de portfólios de Markowitz gera uma performance ruim fora da amostra, devido ao problema dos erros de estimação dos retornos esperados. Para contornar o problema de estimação dos retornos esperados, uma solução comum é ponderar os ativos por meio de um ordenamento. Como também há incerteza no ordenamento dos ativos, os autores 
introduzem um modelo de 'ordenamento robusto', que leva em conta a incerteza do ordenamento na atribuição dos pesos.

Esses métodos apresentam, como característica comum, o estudo da volatilidade histórica dos ativos para gerar as melhores combinações entre os ativos, já que há críticas quanto ao uso de retornos passados na geração de expectativas de retornos futuros. Dentre os mais utilizados na atualidade, destacam-se os chamados métodos de Mínima Variância (MV) e o Equal Weighting (EW).

Cabe observar, adicionalmente, que o método MV não depende de qualquer informação a respeito do retorno esperado dos ativos, gerando assim resultados eficientes e robustos. Entretanto, o MV apresenta problemas quanto à concentração de ativos na carteira, pois foca, exclusivamente, nos ativos com menor risco e maior correlação contrária. Em outras palavras, conforme for a correlação entre os ativos e seu risco, o método pode concentrar a alocação em apenas um ou poucos ativos. Conforme Maillard et al. (2010), a maneira prática de resolver esse problema é atribuir pesos iguais para todos os ativos não livres de risco (Equities) que estarão na carteira. O método de alocação EW, estudado por Choueifaty and Coignard (2008) possui a grande vantagem de ser o único portfólio na fronteira eficiente caso todos os ativos possuírem a mesma correlação, retorno e risco entre si; mas, se os ativos tiverem riscos diferentes, o método pode não ser tão eficiente. Outras carteiras, como a de Mínima Variância, obtiveram retornos maiores e exposições menores ao risco que a carteira formada pelo método exposto (Behr et al., 2013).

Apesar de a teoria sobre alocações em carteira ter evoluído, no sentido de dissipar as incertezas com relação ao retorno esperado, o método de alocação chamado Paridade de Risco (PR) buscou equilibrar risco e retorno (Asness, Frazzini \& Pedersen, 2012; Chaves et al., 2011; Qian, 2011). Os defensores desse método argumentam que, para a criação de uma carteira, a abordagem mais eficiente na alocação de ativos é equalizar os pesos de cada ativo de acordo com sua contribuição ao risco do portfólio, atribuindo-se um peso menor para aqueles ativos mais arriscados e um peso maior para os ativos com volatilidade menor. Tal atribuição de pesos não impede que investidores que desejem um retorno maior, com aversão ao risco menor, não possam alavancar a carteira, mas o portfólio ficará exposto a outros tipos de risco, como risco de liquidez e custo de transação (Asness et al., 2012; Chaves et al., 2011; QIAN, 2011). A alocação leva em consideração somente a covariância das categorias presentes na carteira para que as contribuições de risco destas sejam iguais. Logo, não é necessário assumir retornos futuros como ocorre na maioria das demais estratégias de alocações em carteiras. Conforme declarado por Maillard et al. (2010), o conjunto de títulos resultante é similar a um portfolio de MV com uma restrição de diversificação mínima, e sua volatilidade se encontra entre as volatilidades de portfolios MV e EW.

\subsection{Breve Descrição De Cada Método}

\subsubsection{Mínima Variância}

De acordo com Rubesam e Beltrame (2013), para que se possa implantar, na prática, uma carteira eficiente, é preciso estimar as covariâncias entre os ativos, além de seus retornos médios. Contudo, o erro de estimação, principalmente dos retornos médios, possui um grande impacto na escolha dos pesos, o que faz com que as ações tenham um desempenho ruim nos períodos subsequentes.

Logo, quando o investidor não possui um método preciso para estimar o retorno médio futuro dos ativos (por exemplo, se ele se baseia em médias históricas de retornos), pode ser preferível ignorar completamente as médias e focar na estimação da matriz de covariância, dado haver uma incerteza sobre o retorno esperado de cada ativo, visto que tal retorno depende das escolhas de cada investidor, baseadas nas certezas que os investidores têm sobre o retorno esperado (Rogers et al., , 2007). Neste caso, pode-se determinar apenas uma carteira na chamada fronteira eficiente: a carteira de variância mínima global, ou seja, o ponto mais à esquerda da fronteira eficiente. Conforme Rubesam e Beltrame (2013), a carteira de variância mínima pode ser descrita da seguinte forma: 


$$
\min _{w} L=w^{\prime} \Sigma w,
$$

onde $\Sigma$ é a matriz de covariância dos $\mathrm{n}$ ativos considerados, $\mathrm{w}$ é o vetor dos pesos de cada um dos $\mathrm{n}$ ativos na carteira, $\operatorname{com} \sum_{i=1}^{n} w_{i}=1$ como restrição.

Rubesam e Beltrame (2013) reforçam que, com tal formulação, o método gera resultados como os de um problema denominado de otimização irrestrita, porque os pesos wipodem ter distintos valores, sejam eles positivos ou negativos, com a única condição de que sua soma seja 1 , significando que a carteira deva ser investida integralmente.

Cumpre observar que apenas essa restrição não é o bastante. Para que não haja alavancagem, respeitando-se a premissa de todos os métodos, é necessário impor que os pesos individuais sejam, no mínimo, iguais a zero. Em outras palavras, para que existam posições vendidas, deve-se usar a restrição $\mathrm{w}_{\mathrm{i}} \geq 0$, para todo $\mathrm{i}=1, \ldots, \mathrm{n}$.

Conforme Rubesam e Beltrame (2013), essa restrição adicional é bastante comum nos trabalhos sobre o método MV, gera resultados bastante eficientes, além de ser bastante útil ao lidar com o problema da estimação da matriz de covariância. Por exemplo, se o número de ativos for igual a 100 ( $\mathrm{n}=100)$, há 4950 covariâncias mais 100 variâncias envolvidas na análise, o que em geral é bem maior do que o número de observações, prejudicando a precisão das estimativas. Entretanto, de acordo com Ledoit, Wolf and Honey (2014), um método simples e eficaz para resolver o problema do número de parâmetros na matriz de covariância é restringir as posições vendidas, considerando que os pesos precisam ser maiores ou iguais a zero e, assim, facilitando a implementação numérica do método. Cabe observar, no entanto, que no caso de existência de alavancagem o método pode ser usado, sem perda de generalidade.

\subsubsection{Equal Weighting}

A ideia da alocação EW é deixar a contribuição ao risco de cada ativo igual na formação da carteira; no entanto, uma das hipóteses do modelo é que todos os ativos financeiros possuem a mesma volatilidade (Choueifaty \& Coignard, 2008). Dessa forma, a melhor alocação possível seria atribuir pesos iguais a todos os ativos:

$$
w_{i}=\frac{1}{n}, i=1,2, \ldots, n
$$

onde $\mathrm{W}_{\mathrm{i}}$ é o peso atribuído para cada ativo e $n$ é o número de ativos que irá compor a carteira. Desta forma, gera-se uma carteira com a máxima diversificação possível e com a menor variância global (Choueifaty \& Coignard, 2008).

O método EW também assume que os investidores são racionais, que o mercado é eficiente e que as previsões de volatilidade são precisas, mas apesar de serem premissas clássicas, nem sempre refletem o que ocorre nos mercados, principalmente se há algum choque como uma crise, como foi a que ocorreu no início do século XXI e se refletiu no mercado brasileiro em 2008, porque se todas as previsões de volatilidade dos ativos fossem precisas, não haveria ganhos de curto prazo e o fundo estaria apenas exposto ao prêmio de risco dos ativos.

Se todos os ativos tiverem a mesma correlação, assim como retorno e risco idênticos, a carteira formada pelo método EW será o único portfólio dentro da fronteira eficiente de Markowitz (1952). Portanto, sob certas circunstâncias, o método EW pode trazer resultados melhores quando comparados a outros métodos que são, em sua formação, mais robustos.

\subsubsection{Paridade de risco}


O método de formação de carteiras chamado Paridade de Risco (PR) foi desenvolvido nos últimos dez anos e aplicado nos trabalhos de Maillard et al. (2010), Lee (2011), Qian (2011), Thiagarajan and Schachter (2011) e Clarke, Harindra and Thorley (2013), com descrição dos resultados obtidos nos mercados americano e europeu. A ideia deste método, como o nome sugere, é que cada ativo tenha igual contribuição para o risco total do portfólio, permitindo maior diversificação da carteira, se comparado ao método MV.

A função objetivo adotada neste presente trabalho, considerando-se a crença que para obter maiores retornos esperados é necessário maior risco, é dada por:

$$
\max _{w} L=\sigma_{c}^{2}=w^{\prime} \Sigma w
$$

onde $\sigma_{c}$ é desvio padrão (risco) da carteira, $\Sigma$ é a matriz de covariância dos $\mathrm{n}$ ativos considerados e $\mathrm{w}$ é o vetor dos pesos de cada um dos $\mathrm{n}$ ativos na carteira. Na carteira, por convenção, também se assume que $w_{1}$ representa a fração do patrimônio alocado no ativo livre de risco escolhido, a taxa média de Certificado de Depósito Interbancário (CDI).

As seguintes restrições foram utilizadas para a alocação dos ativos pelo método PR: a primeira refere-se à soma dos pesos de cada um n ativos ser obrigatoriamente igual a $100 \%$ do capital alocado e não permite a existência, em qualquer instante de tempo, da opção da carteira ficar vendida em algum ativo, ou seja,

$$
\sum_{i}^{n} w_{i}=1, \operatorname{com}_{\mathrm{i}} \geq 0,(1)
$$

Para a segunda restrição, define-se S como o limite superior da volatilidade da carteira, que representa o limite de risco máximo que o investidor interessado em montar uma carteira está disposto a tomar, também chamado de grau de diversificação. De acordo com Maillard et al. (2010), o maior grau de diversificação é atingido pelo método EW e o menor grau de diversificação é alcançado pelo método $\mathrm{MV}$, então o valor de $\mathrm{S}$ foi escolhido como igual ao risco do portfólio gerado pelo método EW, de modo a buscar a maior diversificação possível.

Desta forma, a restrição (2) define as contribuições marginais ao risco de forma igualitária para cada um dos $n$ ativos. Por exemplo, se há uma carteira com quatro ativos, todos terão contribuição ao risco exatamente igual à razão entre a volatilidade máxima assumida, $\mathrm{S}$, e o número de ativos, sendo está razão igual 0,25. Vale lembrar que a contribuição ao risco do ativo livre de risco não está presente, pois a contribuição ao risco do CDI é considerada como zero ou, em caso contrário, grande parte do capital disponível seria alocada no ativo livre de risco. Assim, pode-se escrever a restrição como

$$
\frac{w_{i}^{2} \operatorname{Var}\left(R_{i}\right)}{S}=C, \text { para } \mathrm{i}=2, \ldots, \mathrm{n} \text {, isto é, } C=\frac{\operatorname{Diag}(\mathrm{w}) \Sigma \mathrm{w}}{S},(2)
$$

onde $R_{i}$ é o retorno do ativo i e wi é o peso atribuído para cada um dos $n$ ativos, $\Sigma$ é a matriz de covariância dos $n$ ativos considerados, Sé o desvio padrão da carteira contendo os $n$ ativos e Diag(w) é uma matriz diagonal contendo os pesos de cada ativo na diagonal principal, ou seja,

$$
\operatorname{Diag}(w)=\left(\begin{array}{ccc}
w_{1} & \cdots & 0 \\
\vdots & \ddots & \vdots \\
0 & \cdots & w_{n}
\end{array}\right)
$$

\subsection{O impacto de uma crise na alocação de ativos}

De modo geral, a crise econômica afeta as bolsas de valores, ocasionando quedas no valor das ações que se sucedem, trazendo um efeito que varia conforme o grau de concentração e o tamanho da bolsa. No caso brasileiro, a bolsa apresentava, em 2008, quase 400 companhias listadas e uma 
concentração que girava em torno de $40 \%$ em empresas estatais. A crise levou a uma redução no lançamento de ações e no investimento na bolsa e à busca, por parte das empresas, de fontes alternativas de financiamento (Nardes, 2009).

Com relação aos métodos, a literatura demonstra que períodos de crise podem causar as chamadas quebras estruturais em modelos de séries temporais e estas quebras estruturais podem ser ainda mais severas em mercados emergentes como o brasileiro (Garcia \& Ghysels, 1998). A literatura sobre quebra estrutural é extensa quando se trata da estimação de modelos, porém ainda é escassa no Brasil quanto ao impacto que estas quebras estruturais podem trazer à alocação de ativos numa carteira. Alguns autores já trouxeram este tema à tona no mercado brasileiro, como Bortoluzzo et al. (2016) e Sandoval Jr., Bortoluzzo and Venezuela. (2014).

Como destacam Bortoluzzo et al. (2016), há diversas mudanças significativas que podem ocorrer por causa de uma crise, como, por exemplo, queda na liquidez dos ativos, maior volatilidade dos ativos, entre outras. Os autores encontraram evidências de diferenças significativas entre os períodos pré e pós-crise tanto na relação entre os fatores de risco como nos valores e significância dos coeficientes dos três modelos de precificação analisados por eles, conforme citado na seção Fundamento: modelo unifatorial (CAPM) e multifatoriais de risco (modelo de três fatores de Fama e French (1993) e o de quatro fatores de Carhart (1997).

Trazendo esses resultados para a discussão presente neste artigo, uma crise pode provocar mudanças na relação entre as variáveis e os ativos considerados no mercado, impactando diretamente na estimação das variâncias e covariâncias destes ativos. Assim, espera-se que a estrutura de dependência entre os ativos seja diferente antes, depois e durante um período de crise, fazendo com que os resultados dos métodos de Paridade de Risco e Mínima Variância possam apresentar diferenças nestes períodos, uma vez que ambos dependem basicamente da matriz de covariâncias entre os ativos financeiros considerados. Isso instiga à pesquisa sobre o impacto que uma crise pode causar na estruturação da dependência existente entre variáveis e na formação das carteiras.

\section{MÉTODO}

Para realizar a comparação entre os métodos de alocação de ações, coletou-se a base de ativos negociados diariamente na B3 nos anos de 2007 a 2013. O próximo passo foi armazenar, para cada intervalo anual, o excesso de retorno da carteira (diferença entre o retorno da carteira e o retorno do ativo livre de risco), o desvio padrão e o Índice Sharpe dos portfólios (Maillard et al.,2010).

A estimação das matrizes de covariância foi feita utilizando o método amostral, isto é, variâncias e covariâncias sendo calculadas da forma mais simples possível:

$$
S_{X Y}=\sum_{i=1}^{n}\left(X_{i}-\bar{X}\right)\left(Y_{i}-\bar{Y}\right)
$$

Santos e Tessari (2012) argumentam sobre a sensibilidade das carteiras ao método de estimação da matriz de covariâncias, porém, Souza et al. (2017) e Souza et al. (2015), que trabalharam com a formação de carteiras pelos mesmos métodos deste artigo e utilizando dados brasileiros, encontraram similaridade entre os pesos das carteiras com base em seis métodos de estimação das covariâncias, a saber: amostral, decaimento exponencial (EWMA), combinação de dois métodos de decaimento exponencial (modelo Riskmetrics 2006), modelo GARCH, modelo MGARCH e uma combinação não linear de modelos GARCH univariados. Desta forma, neste trabalho optou-se pela estimação da matriz de covariâncias somente utilizando a forma amostral. As empresas que fizeram parte da amostra final estão listadas no Quadro 1 - Conjunto de ativos utilizados para a análise, apresentado no Apêndice A. 
De acordo com Gazotti Junior (2003), o Índice de Sharpe (IS) é uma medida que tem por objetivo avaliar o desempenho de qualquer fundo mediante a relação risco e retorno, já descontando uma taxa de juros livre de risco. Em outras palavras, o IS procura avaliar se o fundo é capaz de gerar retornos excessivos positivos, retornos que condizem à exposição ao risco do investidor. Ele também pode ser usado como alvo de comparação entre outros fundos, já que comparar os fundos diretamente é quase impossível, pois cada fundo possui sua complexidade que o difere dos demais.

O IS é calculado pela razão entre o excesso de retorno da carteira e o desvio padrão da carteira (raiz quadrada da variância):

$$
I S=\frac{R_{c}-R_{f}}{\sigma_{c}}
$$

em que $R_{c}$ é o retorno da carteira de ativos, $R_{\mathrm{f}}$ é o retorno do ativo livre de risco (CDI) e $\sigma_{c}$ é o desvio padrão da carteira.

O numerador fornece informação sobre o desempenho do fundo em relação ao ativo livre de risco, enquanto o denominador fornece informação sobre o risco assumido para ultrapassar o ativo livre de risco. A melhor escolha será pelo fundo que tiver um IS maior e se este valor for negativo significa que teria sido mais vantajoso investir no ativo livre de risco.

Buscou-se analisar o desempenho da formação de carteiras via Paridade de Risco em termos de retorno, volatilidade e Índice de Sharpe, tendo como bases os métodos EW, com diversificação máxima dos ativos na carteira, e MV, com mínima variância. Assim, cada um dos métodos foi executado anualmente do início até o final da amostra dos dados, abrangendo o período de 2007 a 2013. Todos os algoritmos necessários, como métodos de otimização computacional, foram integralmente produzidos no software Matlab.

Antes de detalhar os resultados obtidos, é importante salientar que o Índice de Sharpe (IS) foi utilizado para avaliação do desempenho das carteiras e que ele é, conforme destacam Varga (2001) e Oliveira Filho e Sousa (2015), largamente utilizado no mercado para comparar o desempenho de fundos, pois leva em conta o retorno e o risco dos fundos para geração de uma medida adimensional, que independe das características específicas dos fundos. Ademais, a seleção dos ativos foi feita de acordo com o critério de liquidez diária, sendo considerados todos os ativos negociados diariamente na B3 de 2007 a 2013.

Uma das desvantagens levantadas para o método MV é a pouca diversificação da carteira, uma vez que são alocados apenas ativos com baixa volatilidade, de maneira a minimizar o risco total. Isso pode ser verificado na Tabela 1, que contém o número de ativos considerados para montagem das carteiras em cada um dos anos e quantos destes ativos possuem peso diferente de zero na carteira de MV. Percebe-se que a diversificação das carteiras via MV foi muito baixa, apresentando um máximo de $14,5 \%$ de ativos com peso diferente de zero no ano de 2008 e 2013, o que prejudica a liquidez da carteira do ponto de vista da negociação dos ativos que estão mais concentrados. Por outro lado, a maior concentração poderia levar a um menor gasto de corretagem em operações de compra e venda. As carteiras construídas pelos métodos PR e EW são formadas por todos os ativos disponíveis para aplicação, ou seja, possuem o máximo de diversificação, mas com pesos distribuídos segundo os objetivos de cada método.

Tabela 1 - Número de ativos com 100\% de liquidez diária na B3 e número de ativos com peso diferente de zero na carteira MV para cada ano avaliado, de 2007 a 2013

\begin{tabular}{cccccccc}
\hline & 2007 & 2008 & 2009 & 2010 & 2011 & 2012 & 2013 \\
\hline $\begin{array}{l}\text { Número de ativos com peso dife- } \\
\text { rente de zero na carteira MV }(\%)\end{array}$ & 6 & 8 & 6 & 8 & 5 & 7 & 9 \\
& $(12,5 \%)$ & $(14,5 \%)$ & $(10,7 \%)$ & $(13,3 \%)$ & $(8,2 \%)$ & $(11,3 \%)$ & $(14,5 \%)$ \\
\hline Número total de ativos & 48 & 55 & 56 & 60 & 61 & 62 & 62 \\
\hline
\end{tabular}


Fonte: elaborado pelos autores.

\section{RESULTADOS}

Na Tabela 2 são apresentados os resultados do retorno, risco e IS das carteiras formadas por cada um dos métodos: EW, PR e MV, além do retorno do CDI, considerado como benchmark. Podese considerar que o método PR apresentou resultados intermediários em comparação com os demais métodos no mercado acionário brasileiro, ou seja, assim como o método EW, o PR tem máxima diversificação, mas apresenta risco total um pouco maior que o método $\mathrm{MV}$, como era de se esperar. Resultados similares foram encontrados em Souza et al. (2017) para o Brasil e Maillard et al. (2009), Bruder, Gaussel, Richard and Roncalli (2013) e Lee (2011) para os mercados americano e europeu. No que se refere ao nível de risco das carteiras no período estudado, 2007 a 2013, o método PR também apresentou resultados intermediários em comparação com os métodos EW e MV, isto é:

$\sigma \mathrm{EW} \leq \sigma_{\mathrm{PR}} \leq \sigma_{\mathrm{MV}}$

Tabela 2 - Risco, Retorno e Índice de Sharpe dos três métodos de formação de carteiras: EW, PR e MV para cada ano avaliado, de 2007 a 2013

\begin{tabular}{|c|c|c|c|c|c|c|c|c|}
\hline & Método & 2007 & 2008 & 2009 & 2010 & 2011 & 2012 & 2013 \\
\hline \multirow[t]{3}{*}{ Retorno } & EW & $17.22 \%$ & $-57.20 \%$ & $58.59 \%$ & $5.71 \%$ & $-14.21 \%$ & $7.47 \%$ & $-9.62 \%$ \\
\hline & PR & $14.69 \%$ & $-50.50 \%$ & $52.21 \%$ & $9.38 \%$ & $-4.95 \%$ & $11.11 \%$ & $-4.87 \%$ \\
\hline & MV & $10.90 \%$ & $-41.46 \%$ & $27.29 \%$ & $24.00 \%$ & $7.87 \%$ & $18.61 \%$ & $6.67 \%$ \\
\hline \multirow[t]{3}{*}{ Risco } & EW & $1.61 \%$ & $3.20 \%$ & $1.64 \%$ & $1.13 \%$ & $1.40 \%$ & $1.14 \%$ & $1.11 \%$ \\
\hline & PR & $1.53 \%$ & $2.77 \%$ & $1.09 \%$ & $1.00 \%$ & $1.25 \%$ & $0.91 \%$ & $0.99 \%$ \\
\hline & MV & $1.14 \%$ & $2.65 \%$ & $0.92 \%$ & $0.72 \%$ & $0.71 \%$ & $0.64 \%$ & $0.74 \%$ \\
\hline \multirow[t]{3}{*}{ IS } & EW & 3.35 & -21.74 & 29.71 & -3.56 & -18.44 & -0.82 & -15.93 \\
\hline & PR & 1.88 & -22.70 & 38.84 & -0.35 & -13.24 & 2.98 & -13.06 \\
\hline & MV & -0.81 & -20.32 & 18.93 & 19.83 & -5.26 & 15.95 & -1.88 \\
\hline Retorno & CDI & $11.82 \%$ & $12.38 \%$ & $9.87 \%$ & $9.73 \%$ & $11.60 \%$ & $8.40 \%$ & $8.06 \%$ \\
\hline
\end{tabular}

Em comparação com o ativo livre de risco, o CDI, as carteiras montadas via EW perdem do ativo livre de risco em cinco dos sete anos considerados, enquanto que os outros dois métodos perdem em quatro anos, fato que pode ser explicado pelo período de crise e pelas instabilidades política e econômica que se seguiram.

O IS da carteira montada via MV foi superior em cinco dos sete anos de análise. De 2010 até 2013, o método MV apresentou os melhores resultados e o método PR foi o segundo melhor, assim como encontrado em Souza et al. (2015) e Souza et al. (2017), com a ressalva de que esses autores trabalharam com índices setoriais da B3 como se fossem ativos, enquanto que, neste trabalho, foram utilizadas ações de empresas listadas.

Apesar de o senso comum afirmar que quanto menor o risco, menor será o retorno do fundo, no período estudado o método MV foi soberano, até mesmo em termos de retorno absoluto, em cinco dos sete anos analisados. Adicionalmente, o retorno anual esperado da carteira foi maior (média dos retornos anuais) e a carteira MV foi a única a apresentar retorno positivo no período total. Para cada $\mathrm{R} \$ 1,00$ (um real) investido em janeiro de 2007 na carteira MV, o investidor resgataria, em dezembro de 2013, R \$1,40 (um real e quarenta centavos) em termos nominais, enquanto que aplicações nas carteiras EW e PR gerariam perdas de 30\% e 5\%, respectivamente, também em termos nominais.

Para ilustrar a análise, a Figura 1 contém três gráficos para comparar os métodos de alocação de carteira de acordo com o IS, risco e retorno de cada um deles, visando identificar qual dos métodos apresentou o maior retorno e o menor risco ao longo do tempo. 
Figura 1. Índice de Sharpe, risco e retorno dos três métodos de formação de carteiras: EW, PR e MV, para cada ano avaliado de 2007 a 2013.

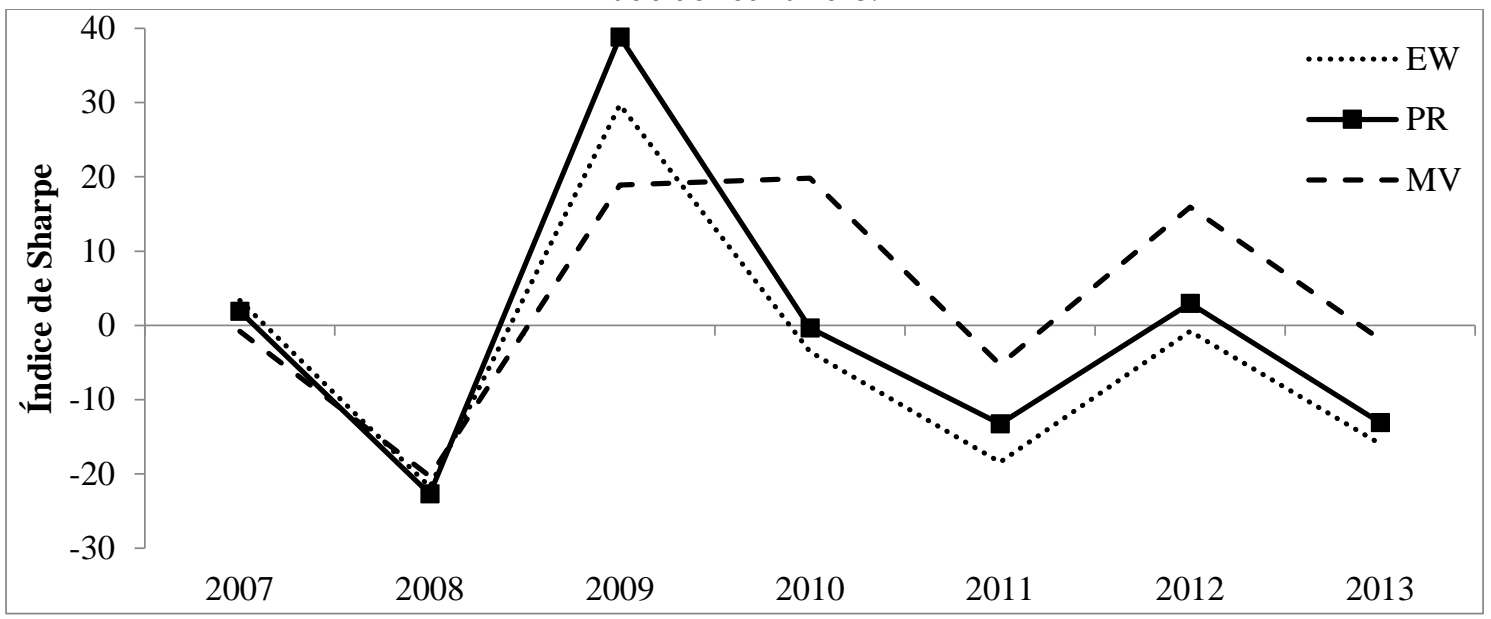

a. Índice de Sharpe

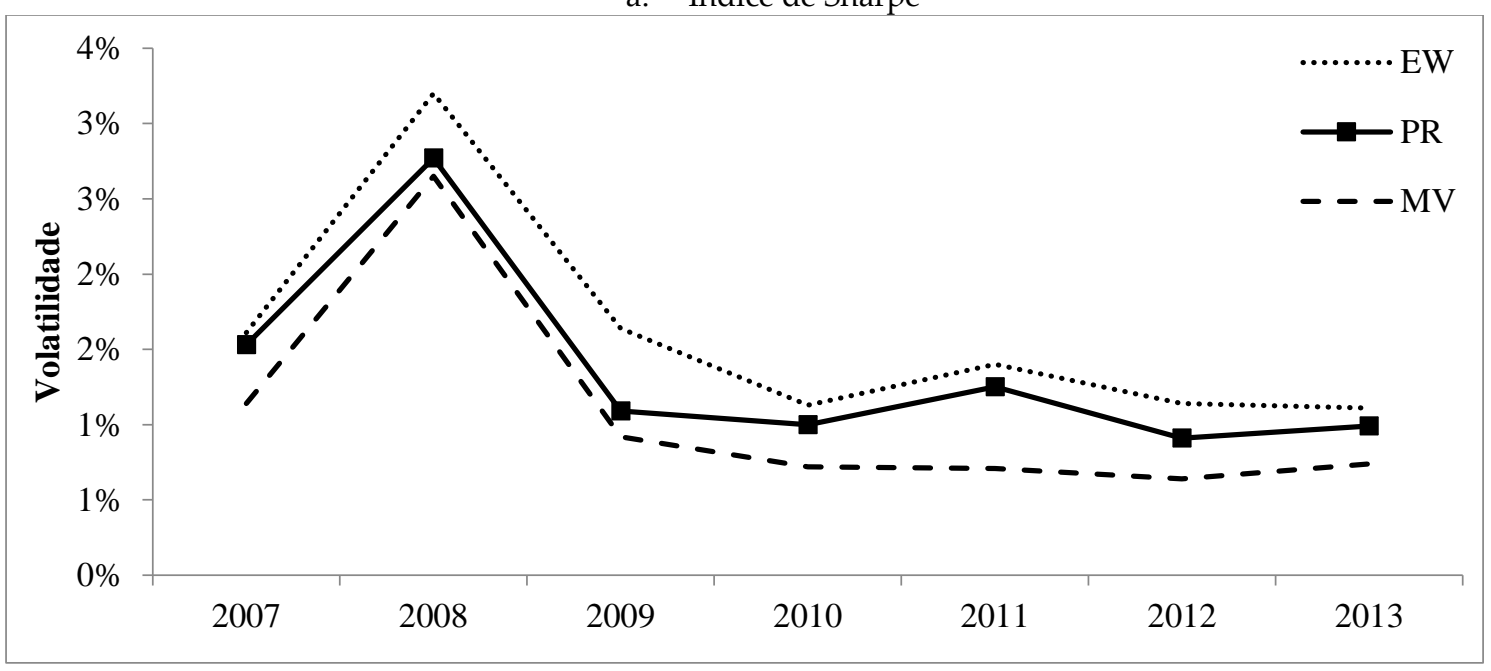

b. Risco

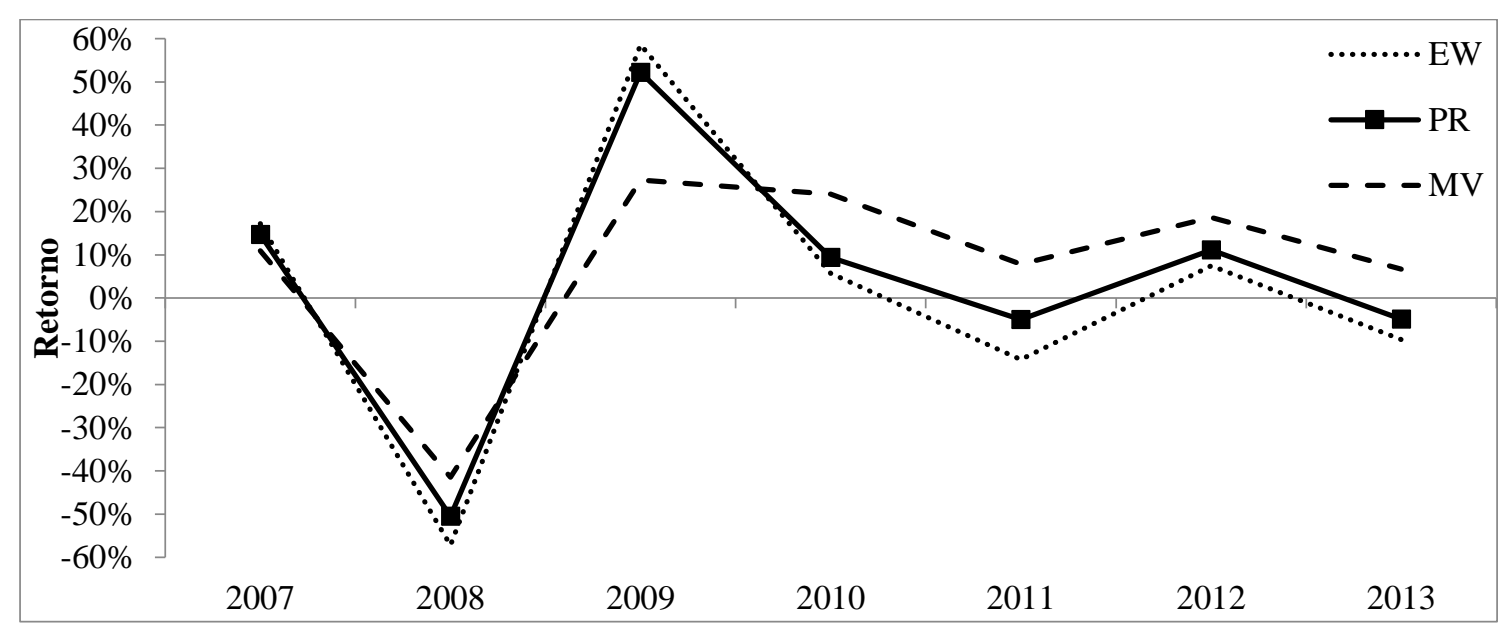

c. Retorno

Fonte: elaborado pelos autores. 
Analisando-se o gráfico (a) da Figura 1 é possível concluir que o método MV oferece uma melhor relação risco retorno após a turbulência da crise do subprime e em tempos de expectativas negativas em que ocorreu a crise política e econômica no país (2010 a 2013), o que provavelmente aconteceu porque as carteiras formadas por MV possuem participação de poucos ativos do mercado, sendo estes ativos aqueles com menor risco e maior retorno no período. Já o método PR apresentou melhor resultado em tempos de agitação do mercado (2009), quando a crise financeira mundial realmente chegou ao Brasil. A explicação para este resultado está no fato de o método PR controla a participação de cada ativo no portfólio de acordo com a sua volatilidade, permitindo carteiras com maior diversificação do que o método MV e, assim, com risco quase mínimo (volatilidade das carteiras por PR e MV são similares na Figura 1(b), enquanto o retorno da carteira via PR é maior do que a de MV na Figura 1 (c)).

No ano que precedeu à crise financeira do subprime (2007), período de euforia do mercado brasileiro, o método EW foi o melhor, o que demonstra que em períodos de calmaria e euforia de mercado, compor uma carteira com todos os ativos tendo mesmo peso, pode ser uma estratégia simples e rentável. Comparando o método EW com o método PR na Figura 1 (a), pode-se perceber que o método PR apresenta maior IS em praticamente todo o tempo da amostra, exceto em 2007 e 2008 em que os métodos apresentam valores similares, o que pode ser um indicativo para estudos futuros, visando identificar outros métodos que consigam tratar melhor a volatilidade dos ativos.

\section{CONCLUSÃO}

O objetivo desse trabalho foi realizar uma comparação do desempenho de carteiras montadas de acordo com os métodos Paridade de Risco (PR), Mínima Variância (MV) e Equal Weighting (EW) no mercado brasileiro em períodos pré, pós e durante a crise internacional de 2008. Devido aos três métodos estudados serem métodos diferentes em suas formações, torna-se oportuno notar o seu comportamento nos períodos estudados, especialmente em 2008, na eclosão da crise nos EUA. Constata-se que os métodos PR e EW podem destacar-se mais em períodos de queda na aversão ao risco, mas em tempos de estabilidade não se pode afirmar que tais métodos possam entregar resultados mais satisfatórios que o método $\mathrm{MV}$.

$\mathrm{Na}$ amostra pesquisada, o método MV obteve o maior retorno e menor risco, apresentando o maior IS dentre os métodos analisados, além ter tido a única carteira a apresentar retorno efetivo positivo no período analisado. Estes resultados vão ao encontro do trabalho de Souza et al. (2017), mas diferem dos encontrados em Chaves et al. (2011), em que o método PR apresentou o melhor desempenho.

Apesar do melhor desempenho das carteiras montadas pelo método MV, estas carteiras possuem poucos ativos em sua formulação, apresentando muita concentração em poucos ativos, isto é, são menos diversificadas do que as carteiras formadas pelos métodos EW e PR.

Assim como obtido no Brasil com o uso de índices setoriais em Souza et al. (2017) e nos mercados estrangeiros em Maillard et al. (2009), Bruder et al. (2013) e Lee (2011), os resultados com os ativos brasileiros demonstram que o método PR leva à obtenção de carteiras intermediárias, em comparação com os métodos MV e EW. As carteiras formadas via PR são mais diversificadas do que as formadas via MV e tão diversificadas quanto aquelas obtidas via $\mathrm{EW}$, mas com pesos que permitem com que o risco via PR seja inferior ao risco das carteiras formadas via EW.

Apesar de os dados mostrarem que o método MV conseguiu se sobressair entre os demais (EW e PR) nos períodos de incerteza e pós-crise, não se pode afirmar de maneira definitiva que qualquer método é superior aos demais.

Uma limitação do trabalho foi a adoção da premissa de não existência de posições vendidas, o que pode ter influenciado o resultado final. Por sua formulação, o método PR obrigatoriamente investirá em todos os ativos que compõem a carteira, mesmo aqueles que apresentam 
alta volatilidade, mas se fosse possível adotar posições vendidas, talvez esses ativos pudessem ser usados para obtenção de caixa e, assim, alavancar a carteira nos ativos mais seguros e de qualidade. Ademais, o exercício de Chaves et al. (2011) envolveu um número maior de ativos, além de ter sido feito com uma amostra temporal maior e em um mercado de maior volume financeiro e de economia mais estável. Consequentemente, sugere-se para pesquisas futuras o estudo de uma janela temporal maior, em que o prêmio de risco de mercado tenha se apresentado positivo. Também sugerimos, em pesquisas futuras, a inclusão de mais ativos financeiros entre os disponíveis para a formação das carteiras.

\section{REFERÊNCIAS}

Araújo, A.C. de, \& Montini, A. de A. (2015). Análise de métricas de risco na otimização de portfolios de ações. Revista de Administração, 50(2), 208-228.

Asness, C.S., Frazzini, A., \& Pedersen, L.H. (2012). Leverage Aversion and Risk Parity. Financial Analyst Journal, 68(1), 47-59.

Behr, P., Guettler, A. \& Miebs, F. (2013). On portfolio optimization: Imposing the right constraints. Journal of Banking \& Finance, 37(4), 1232-1242.

Bortoluzzo, A. B., Venezuela, M. K., Bortoluzzo, M. M., \& Nakamura, W. T. (2016). Influência da crise financeira de 2008 na previsibilidade dos modelos de apreçamento de ativos de risco no Brasil. Revista Contabilidade E Finanças, 27(72), 408-420.

Bruder, B., Gaussel, N., Richard, J-C; \& Roncalli, T. Regularization of Portfolio Allocation. June 2013. White Paper Issue \# 10 (Lyxor Research). Retrieved Dec 20, 2016, from http://www.lyxor.com/uploads/tx_bilyxornews/Regularization_of_portfolio_allocation.pdf.Chaves, D., Hsu, J., Li, F., \& Shakernia, O. (2011). Risk Parity Portfolio VS. Other Asset Allocation Heuristic Portfolio assets. The Journal of Investing, 20(1), 108-118.

Carhart, M. M. (1997). On persistence in mutual fund performance. The Journal of Finance, 52, 57-82.

Choueifaty, Y., \& Coignard, Y. (2008). Toward maximum diversification. Journal of Portfolio Management, 35(1), 40.

Chow, T. M., Hsu, J., Kalesnik, V., \& Little, B. (2011). A survey of alternative equity index strategies. Financial Analysts Journal, 67(5), 37-57.

Clarke, R., Harindra, S., \& Thorley, S. (2013). Risk Parity, Maximum Diversification, and Minimum Variance: An Analytic Perspective. The Journal of Portfolio Management, 39(3), 39-53.

Fabozzi, F.J., Gupta, F., \& Markowitz, H.M. (2002). The legacy of Modern Portfolio Theory. The Journal of Investing, 11(3), 7-22.

Fama, E. F., \& French, K. R. (1993). Common risk factors in the returns on stocks and bonds. Journal of Financial Economics, 33, 3-56.

Ferri, R.A. All about asset allocation. 2ª ed. São Paulo: Mc Graw Hill Interamericana do Brasil, 2010.

Fliege, J., \& Werner, R. (2014). Robust multiobjective optimization e applications in portfolio optimization. European Journal of Operational Research, 234(2), 422-433.

Garcia, R., \& Ghysels, E. (1998). Structural change and asset pricing in emerging markets. Journal of International Money and Finance, 17(3), 455-473. 
Gazotti Junior, I. (2003). Conceitos de VaR e Índice de Sharpe para Leigos. Disclosure das Transações Financeiras. Ano VIII, edição 92. Retrieved Dec 20, 2016, from http://www.webfinder.com.br/disclosure/PDF/dis-92-3.pdf

Leal, R. P. C., Silva, A. L. C., \& Ribeiro, T. S. (2002). Alocação Ótima de Ativos em Fundos de Pensão Brasileiros. Relatório Técnico Coppead n³51. Disponível em: <http://www.coppead.ufrj.br/upload/publicacoes/351.pdf.>.

Ledoit, O., Wolf, M., \& Honey, I. (2004) Shrunk the Sample Covariance Matrix. The Journal of Portfolio Management, 30(4), 110-119.

Lee, W. (2011). Risk-Based Asset Allocation: A New Answer to an Old Question? The Journal of Portfolio Management, 37(4),11-28.

Levy, H., \& Levy, M. (2014). The benefits of differential variance-based constraints in portfolio optimization. European Journal of Operational Research, 234(2), 372-381.

Maillard, S., Roncalli, T., \& Teïletche, J. (2010). The properties of equally weighted risk contribution portfolios. The Journal of Portfolio Management, 36(4), 60-70.

Markowitz, H. (1952). Portfolio selection. The journal of finance, 7(1), 77-91.

Nardes, A. Relatório e pareceres prévios sobre as contas do governo da República. Exercício de 2008. Brasília: TCU, 2009.

Nguyen, T. D., \& Lo, A. W. (2012). Robust ranking and portfolio optimization. European Journal of Operational Research, 221(2), 407-416.

Oliveira Filho, B. G., \& Sousa, A.F. (2015). Fundos de investimento em ações no Brasil: métricas para avaliação de desempenho. REGE, 22(1), 61-76.

Qian, E. (2011). Risk Parity and Diversification. The Journal of Investing, 20(1), 119-127.

Rogers, P., Securato, J. R., \& Ribeiro, K. C. S. (2007). Finanças Comportamentais no Brasil: Um Estudo Comparativo. Revista de Economia e Administração, 6(1), 49-68.

Rubesam, A., \& Beltrame, A. L. (2013). Carteiras de Variância Mínima no Brasil, Rev. Bras. Finanças (online), 11(1), 81-118.

Sandoval Jr, L., Bortoluzzo, A. B., \& Venezuela, M. K. (2014). Not all that glitters is RMT in the forecasting of risk of portfolios in the Brazilian stock market. Physica A, 410, 94-109.

Santos, A. A. P., \& Tessari, C. (2012). Técnicas quantitativas de otimização de carteiras aplicadas ao mercado de ações brasileiro. Revista Brasileira de Finanças, 10 (3), 369-394.

Sharpe, W. F. (1963). A Simplified Model of Portfolio Analysis. Managent Science, 9(2), 277-293.

. (1964). Capital Asset Prices: A theory of market equilibrium under conditions of risk. Journal of Finance, 19(3), 425-442.

Souza, P. O., Filomena, T. P., Caldeira, J. F., Borenstein, D., \& Righi M. B., (2017). Risk parity in the Brazilian market. Economics Bulletin, 37(3), 1555-1566.

Souza, P. O., Filomena, T. P., \& Caldeira, J. F. (2015). A paridade de risco no cenário brasileiro. Encontro da ANPEC. Retrieved Dec 20, 2016, from http://www.anpec.org.br/encontro/2015/submissao/files_I/i8-1bf44991291d07d3b82b308025451b7c.pdf

Thiagarajan, S. R., \& Schachter, B. (2011). Risk Parity: Rewards, Risks, and Research Opportunities. The Journal of Investing, 20(1), 79-89. 
Varga, G. (2001). Índice de Sharpe e outros indicadores de performance aplicados a fundos de ações brasileiros. Revista de Administração Contemporânea, 5(3), 215-245.

Zhou, G. (2009). Beyond Black-Litterman: letting the data speak. The Journal of Portfolio Management, 36(1), 36-45. 


\section{APÊNDICE A}

Quadro 1 -Conjunto de ativos utilizados para a análise.

\begin{tabular}{|c|c|c|c|c|c|c|c|}
\hline Empresa listada & 2007 & 2008 & 2009 & 2010 & 2011 & 2012 & 2013 \\
\hline ABEV3 & $x$ & $x$ & $x$ & $X$ & $\mathrm{X}$ & $\mathrm{X}$ & $X$ \\
\hline ALLL3 & $X$ & $X$ & $X$ & $X$ & $X$ & $X$ & $X$ \\
\hline BBAS3 & $X$ & $X$ & $X$ & $X$ & $X$ & $X$ & $X$ \\
\hline BBDC3 & $x$ & $X$ & $X$ & $X$ & $x$ & $x$ & $X$ \\
\hline BBDC4 & $x$ & $X$ & $X$ & $X$ & $X$ & $X$ & $X$ \\
\hline BISA3 & $x$ & $x$ & $X$ & $x$ & $x$ & $x$ & $X$ \\
\hline BRAP4 & $x$ & $X$ & $X$ & $X$ & $X$ & $x$ & $X$ \\
\hline BRFS3 & $x$ & $x$ & $X$ & $X$ & $X$ & $x$ & $X$ \\
\hline BRKM5 & $x$ & $X$ & $X$ & $x$ & $X$ & $x$ & $X$ \\
\hline CCRO3 & $X$ & $X$ & $X$ & $X$ & $X$ & $X$ & $X$ \\
\hline CESP6 & $x$ & $X$ & $X$ & $X$ & $X$ & $X$ & $X$ \\
\hline CMIG4 & $X$ & $X$ & $X$ & $X$ & $X$ & $X$ & $X$ \\
\hline CPFE3 & $x$ & $x$ & $X$ & $X$ & $x$ & $x$ & $x$ \\
\hline CPLE6 & $x$ & $x$ & $X$ & $X$ & $x$ & $x$ & $X$ \\
\hline CRUZ3 & $x$ & $x$ & $X$ & $X$ & $x$ & $x$ & $x$ \\
\hline CSAN3 & $X$ & $X$ & $X$ & $X$ & $x$ & $X$ & $X$ \\
\hline CSNA3 & $X$ & $x$ & $X$ & $X$ & $x$ & $x$ & $X$ \\
\hline CYRE3 & $X$ & $X$ & $X$ & $X$ & $X$ & $x$ & $X$ \\
\hline ELET3 & $x$ & $X$ & $X$ & $X$ & $x$ & $x$ & $X$ \\
\hline ELET6 & $x$ & $X$ & $X$ & $X$ & $X$ & $x$ & $X$ \\
\hline ELPL4 & $x$ & $x$ & $X$ & $X$ & $x$ & $x$ & $X$ \\
\hline EMBR3 & $x$ & $X$ & $X$ & $X$ & $X$ & $X$ & $X$ \\
\hline ENBR3 & $X$ & $X$ & $X$ & $X$ & $X$ & $X$ & $X$ \\
\hline GFSA3 & $X$ & $X$ & $X$ & $X$ & $X$ & $X$ & $X$ \\
\hline GGBR4 & $x$ & $X$ & $X$ & $x$ & $x$ & $X$ & $X$ \\
\hline GOAU4 & $x$ & $X$ & $X$ & $X$ & $x$ & $x$ & $X$ \\
\hline GOLL4 & $X$ & $x$ & $X$ & $X$ & $X$ & $X$ & $X$ \\
\hline ITSA4 & $x$ & $X$ & $X$ & $X$ & $X$ & $X$ & $X$ \\
\hline ITUB4 & $x$ & $X$ & $X$ & $x$ & $x$ & $X$ & $X$ \\
\hline LAME4 & $X$ & $X$ & $X$ & $X$ & $X$ & $X$ & $X$ \\
\hline LIGT3 & $X$ & $X$ & $X$ & $X$ & $X$ & $X$ & $X$ \\
\hline LREN3 & $X$ & $X$ & $X$ & $X$ & $X$ & $x$ & $X$ \\
\hline NATU3 & $X$ & $X$ & $X$ & $X$ & $X$ & $X$ & $X$ \\
\hline OIBR4 & $x$ & $X$ & $X$ & $X$ & $x$ & $X$ & $X$ \\
\hline PCAR4 & $x$ & $x$ & $x$ & $x$ & $x$ & $x$ & $x$ \\
\hline PETR3 & $x$ & $x$ & $X$ & $x$ & $x$ & $x$ & $x$ \\
\hline PETR4 & $X$ & $X$ & $X$ & $X$ & $X$ & $X$ & $X$ \\
\hline RENT3 & $X$ & $X$ & $X$ & $X$ & $X$ & $X$ & $X$ \\
\hline RSID3 & $X$ & $X$ & $X$ & $X$ & $X$ & $X$ & $X$ \\
\hline SBSP3 & $x$ & $x$ & $X$ & $X$ & $X$ & $X$ & $X$ \\
\hline SUZB5 & $x$ & $X$ & $X$ & $X$ & $X$ & $X$ & $X$ \\
\hline TBLE3 & $X$ & $X$ & $X$ & $X$ & $X$ & $X$ & $X$ \\
\hline TIMP3 & $x$ & $X$ & $X$ & $X$ & $X$ & $X$ & $X$ \\
\hline UGPA3 & $X$ & $X$ & $X$ & $X$ & $X$ & $X$ & $X$ \\
\hline USIM5 & $X$ & $X$ & $X$ & $X$ & $X$ & $X$ & $X$ \\
\hline VALE3 & $X$ & $X$ & $X$ & $x$ & $X$ & $X$ & $X$ \\
\hline VALE5 & $x$ & $X$ & $X$ & $X$ & $X$ & $X$ & $x$ \\
\hline VIVT4 & $x$ & $X$ & $X$ & $X$ & $X$ & $X$ & $x$ \\
\hline BRML3 & & $X$ & $x$ & $X$ & $x$ & $X$ & $x$ \\
\hline EVEN3 & & $x$ & $x$ & $X$ & $x$ & $X$ & $x$ \\
\hline JBSS3 & & $X$ & $X$ & $X$ & $X$ & $x$ & $X$ \\
\hline
\end{tabular}




\begin{tabular}{|l|l|l|l|l|l|l|l|}
\hline MRFG3 & & $\mathrm{X}$ & $\mathrm{X}$ & $\mathrm{X}$ & $\mathrm{X}$ & $\mathrm{X}$ & $\mathrm{X}$ \\
\hline MRVE3 & & $\mathrm{X}$ & $\mathrm{X}$ & $\mathrm{X}$ & $\mathrm{X}$ & $\mathrm{X}$ & $\mathrm{X}$ \\
\hline PDGR3 & & $\mathrm{X}$ & $\mathrm{X}$ & $\mathrm{X}$ & $\mathrm{X}$ & $\mathrm{X}$ & $\mathrm{X}$ \\
\hline MMXM3 & & $\mathrm{X}$ & & & & & \\
\hline BVMF3 & & & $\mathrm{X}$ & $\mathrm{X}$ & $\mathrm{X}$ & $\mathrm{X}$ & $\mathrm{X}$ \\
\hline HYPE3 & & $\mathrm{X}$ & $\mathrm{X}$ & $\mathrm{X}$ & $\mathrm{X}$ & $\mathrm{X}$ \\
\hline CIEL3 & & & $\mathrm{X}$ & $\mathrm{X}$ & $\mathrm{X}$ & $\mathrm{X}$ \\
\hline CTIP3 & & & $\mathrm{X}$ & $\mathrm{X}$ & $\mathrm{X}$ & $\mathrm{X}$ \\
\hline FIBR3 & & & $\mathrm{X}$ & $\mathrm{X}$ & $\mathrm{X}$ & $\mathrm{X}$ \\
\hline SANB11 & & & $\mathrm{X}$ & $\mathrm{X}$ & $\mathrm{X}$ & $\mathrm{X}$ \\
\hline BRPR3 & & & & & $\mathrm{X}$ & $\mathrm{X}$ & $\mathrm{X}$ \\
\hline QUAL3 & & & & & & $\mathrm{X}$ & $\mathrm{X}$ \\
\hline
\end{tabular}

Fonte: elaborado pelos autores. 